\title{
Effects of Laminaria japonica polysaccharides on exercise endurance and oxidative stress in forced swimming mouse model
}

\author{
Feiwei Yan ${ }^{1 *}$ and Haitao Hao ${ }^{2}$
}

\begin{abstract}
Background: Polysaccharides are the major active ingredients responsible for the bioactivities of Laminaria japonica. However, the effects of L. japonica polysaccharides (LJP) on exercise endurance and oxidative stress have never been investigated. Therefore, this study was conducted to investigate the effects of LJP on exercise endurance and oxidative stress in a forced swimming mouse model. The animals were divided into four groups, namely the control (C), LJP-75, LJP-150, and LJP-300 groups, which received physiological saline and 75, 150, and $300 \mathrm{mg} \mathrm{kg}^{-1} \mathrm{LJP}_{\text {, respec- }}$ tively, by gavage once a day for 28 days. This was followed by a forced swimming test and measurements of various biochemical parameters.
\end{abstract}

Results: LJP increased swimming time to exhaustion, the liver and muscle glycogen content, and levels of superoxide dismutase, glutathione peroxidase, and catalase in the serum, liver, and muscle, which were accompanied by corresponding decreases in the malondialdehyde (MDA) content of the same tissues. Furthermore, decreases in blood lactic acid and serum myeloperoxidase (MPO) levels were observed.

Conclusion: LJP enhanced exercise endurance and protected mice against exhaustive exercise-induced oxidative stress.

Keywords: Laminaria japonica polysaccharides, Forced swimming test, Exercise endurance, Oxidative stress, Mice

\section{Background}

The generation of reactive oxygen species (ROS) is a necessary and unavoidable consequence of aerobic metabolism [1]. The enhanced oxygen consumption during exercise leads to an increased flux of oxygen through the mitochondria, and $2-5 \%$ of this oxygen is not completely reduced to water and, therefore, generates ROS [2]. Under normal physiological conditions, cells have adequate defenses against ROS production and enough endogenous enzymatic and nonenzymatic antioxidant reserves $[3,4]$. However, during strenuous physical exercise, the rate of ROS generation exceeds that of their removal

\footnotetext{
*Correspondence: yanzjfeiwei@163.com

1 Public PE Department, Zhejiang Shuren University, No. 8 Shuren Street, Hangzhou 310015, Zhejiang Province, People's Republic of China Full list of author information is available at the end of the article
}

and oxidative stress occurs [5]. Consequently, accumulated excessive ROS can attack vital biomolecules such as plasma membrane lipids and proteins and, thereby, deteriorate normal cellular functions and further contribute to muscle damage [6]. Specifically, it has been shown that strenuous physical exercise decreases antioxidants levels and increases lipid peroxidation markers in the blood and tissues [7]. Therefore, antioxidant supplementation may protect against exhaustive exercise-induced oxidative stress by forming less active radicals or quenching free radicals and ROS [8]. Many antioxidant bioactive compounds, such as polysaccharides from Radix pseudostellariae [Pseudostellaria heterophylla (Miq.) Pax], polysaccharides from Auricularia auricula, polysaccharides from Cordyceps sinensis mycelium, polysaccharides from Ganoderma lucidum, salidroside, ginsenoside- $\operatorname{Rg}_{1}$, 
ginsenoside- $R b_{1}$, flavonoid from Citrus limon (L.) Burm. F. as well as polyphenols from Vaccinium corymbosum L., have been reported for their protective effects on exhaustive exercise-induced oxidative stress [4, 9-16].

The brown seaweed Laminaria japonica, is a common seafood consumed in China and numerous other countries and has been documented as a drug in traditional Chinese medicine (TCM) [17]. In the ancient literature, L. japonica has been recorded as an important therapeutic agent for phlegm elimination, detumescence, and weight loss for more than 1000 years [18]. Over the past decades, L. japonica has been the focus of attention of chemists and pharmacologists because of its abundant functional compound content and the associated biological properties. The major active constituents of $L$. japonica are polysaccharides including alginate, fucoidan, and laminarin [19]. Recent studies have demonstrated that $L$. japonica polysaccharides (LJP) have a wide range of biological properties including anti-apoptosis, antivirus, anticoagulant, antitumor, antithrombotic, anti-radiation, hypoglycemic, hypolipidemic, and immunostimulatory [20-23]. Furthermore, LJP protected endogenous antioxidant enzymes, inhibited lipid peroxidation, and exhibited high antioxidant activities including the oxygen radical absorbance capacity (ORAC), 2,2'-azino-bis-(3-ethylbenzthiazoline-6sulfonic acid) (ABTS) and reduced power tests [24, 25], suggesting that LJP might reduce exhaustive exercise-induced oxidative stress. Therefore, the current study aimed to demonstrate the protective effects of LJP against exercise endurance and oxidative stress in a forced swimming mouse model.

\section{Results and discussion}

\section{Effects of LJP on swimming time to exhaustion of mice}

Exercise endurance is an important parameter for evaluating anti-fatigue treatments, and the forced swimming test has been widely used for this purpose with high reproducibility [26]. The lengths of the swimming time to exhaustion indicate the degree of exercise tolerance and fatigue. As shown in Fig. 1, swimming time to exhaustion of the LJP-75, LJP-150, and LJP-300 groups were significantly longer than that of the control (C) group $(p<0.05)$ with increased rates of $30.58,45.57$, and $51.72 \%$, respectively. This result indicates that LJP enhanced the exercise endurance and had anti-fatigue effects.

\section{Effects of LJP on blood lactic acid levels of mice}

The blood lactic acid (BLA) level tends to increase during strenuous physical exercise because anaerobic metabolism becomes the dominant energy-producing mechanism. The increased lactic acid level further reduces the $\mathrm{pH}$, which could induce various biochemical and physiological effects including glycolysis, phosphofructokinase, and calcium ion release through muscular contraction [27]. Therefore, BLA is an important marker

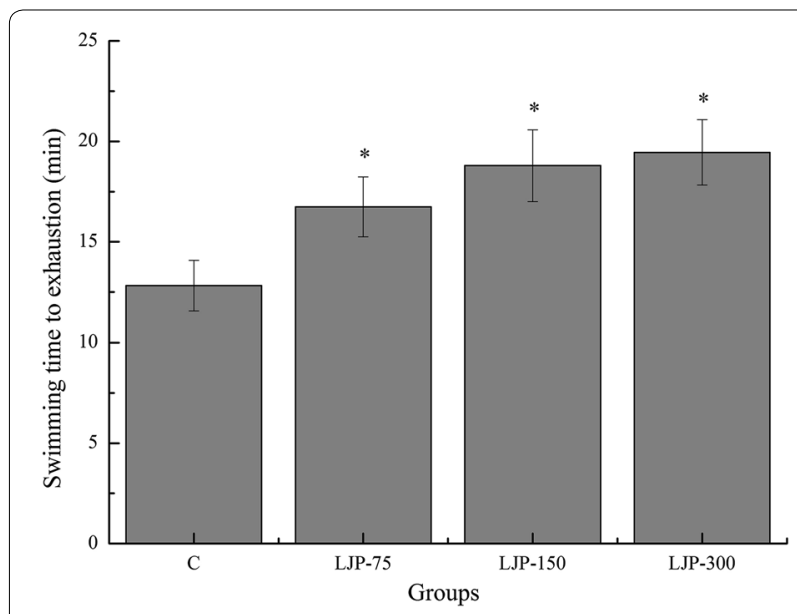

Fig. 1 Effects of L.japonica polysaccharides on swimming time to exhaustion of mice. Data are mean \pm standard deviation (SD); $n=12$, ${ }^{*} p<0.05$ compared with control (C) group

for evaluating the degree of fatigue of a living organism. As shown in Fig. 2, BLA levels of the LJP-75, LJP-150, and LJP-300 groups were significantly lower than that of the $\mathrm{C}$ group $(p<0.05)$, and the decrease rates were 25.99 , 35.65 , and $52.58 \%$, respectively. This result indicates that LJP effectively inhibited and lowered BLA production and, thereby, retarded the occurrence of fatigue.

\section{Effects of LJP on glycogen contents of the liver and muscle of mice}

Energy for exercise is derived initially from the breakdown of glycogen in muscle which may be depleted during strenuous exercise, and at later stages, the energy is derived from the liver glycogen $[10,28]$. The depletion of

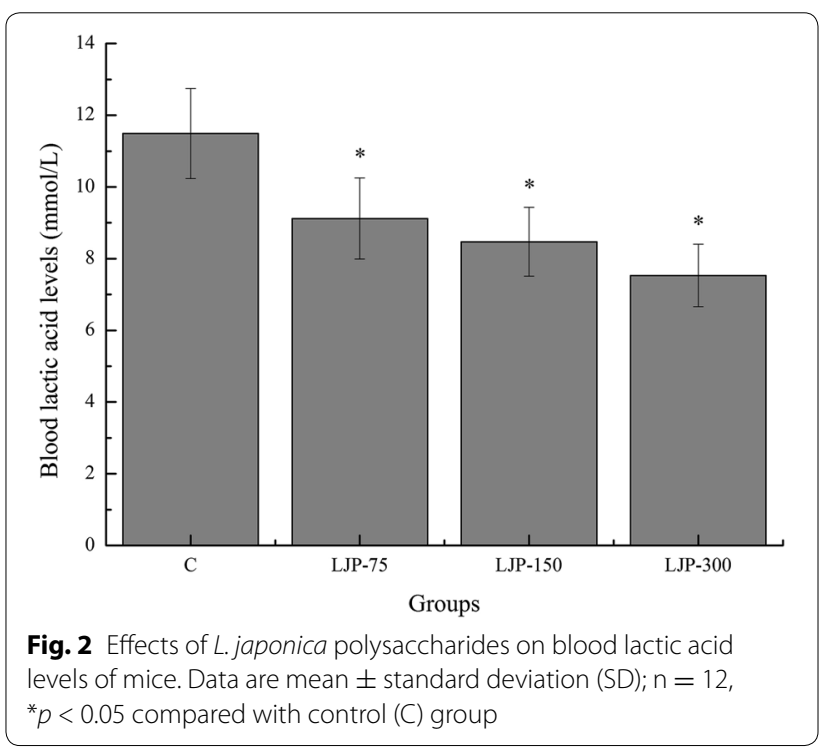


liver glycogen leads to the onset of fatigue [12]. As shown in Fig. 3, the glycogen contents of the liver and muscles of the LJP-75, LJP-150, and LJP-300 groups were significantly higher than that of the $\mathrm{C}$ group $(p<0.05)$. Specifically, the increased rates in the liver were $34.73,61.19$, and $93.76 \%$, respectively, while in muscle, $15.29,25.48$, and $32.48 \%$, respectively. The results indicate that LJP reduced the liver and muscle glycogen consumption by improving its reserve or reducing the consumption during exercise. Furthermore, the anti-fatigue effects of LJP might be related to the improved metabolic control of exercise and activation of energy metabolism [29].

\section{Effects of LJP on superoxide dismutase (SOD), glutathione peroxidase (GPX), and catalase (CAT) levels of mouse serum, liver and muscle}

It was previously demonstrated that antioxidant enzymes play a significant role in protecting the body against ROS [15]. The principal antioxidant enzymes include superoxide dismutase (SOD), glutathione peroxidase (GPx), and catalase (CAT), and they act to reduce ROS [30]. Regular physical exercise has been shown to increase antioxidant enzyme activities in the blood and tissues of humans and animals [31, 32], which can be attributed to a compensatory response to counteract the possible detrimental effects associated with oxidative stress [33]. However, other studies have reported that strenuous exercise causes a dramatic drop in antioxidant enzyme activities in the blood and tissues [14]. Although inconsistent findings have been reported on the level of antioxidant enzymes, it appears that the variation of these enzymes is dependent not only on the type of tissues measured but also on the mode and intensity of exercise [15].

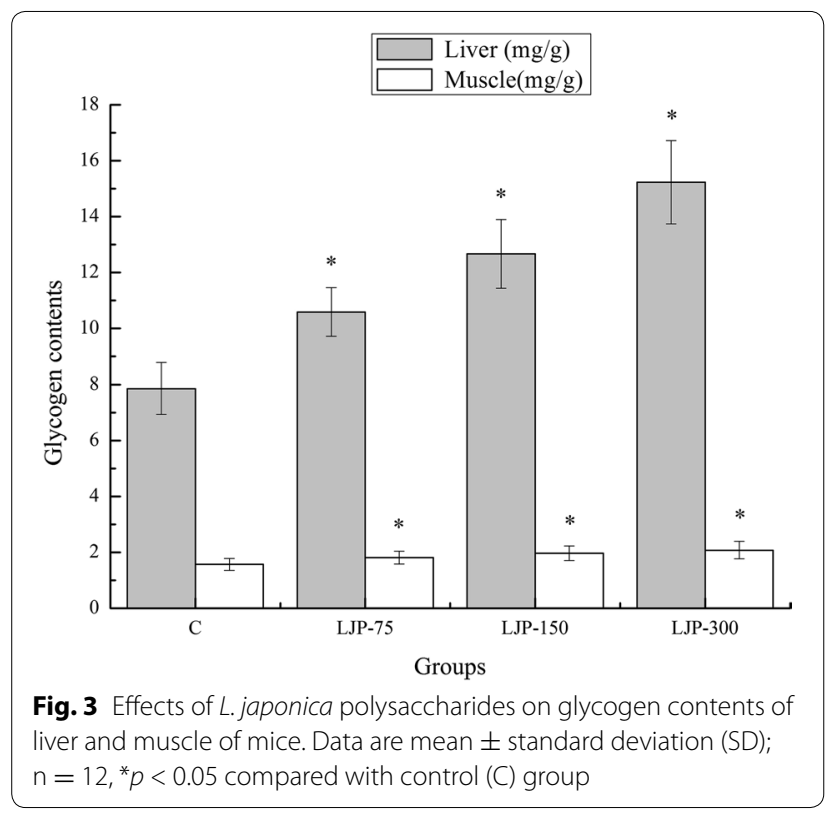

Previous studies have demonstrated that the variation in the levels of antioxidant enzymes in different tissues might be due to tissue-specific metabolic differences [34]. The liver is a critical physiological metabolic organ in organisms, involved in almost all of the substance metabolism, and contains higher levels of antioxidant enzymes than other tissues, which in turn release more ROS with increased lipid peroxidation products $[35,36]$. Recent studies have demonstrated a tissue-specific expression of GPx and CAT, with their highest activities occurring in the liver $[4,12,34]$. As shown in Fig. 4a, SOD serum

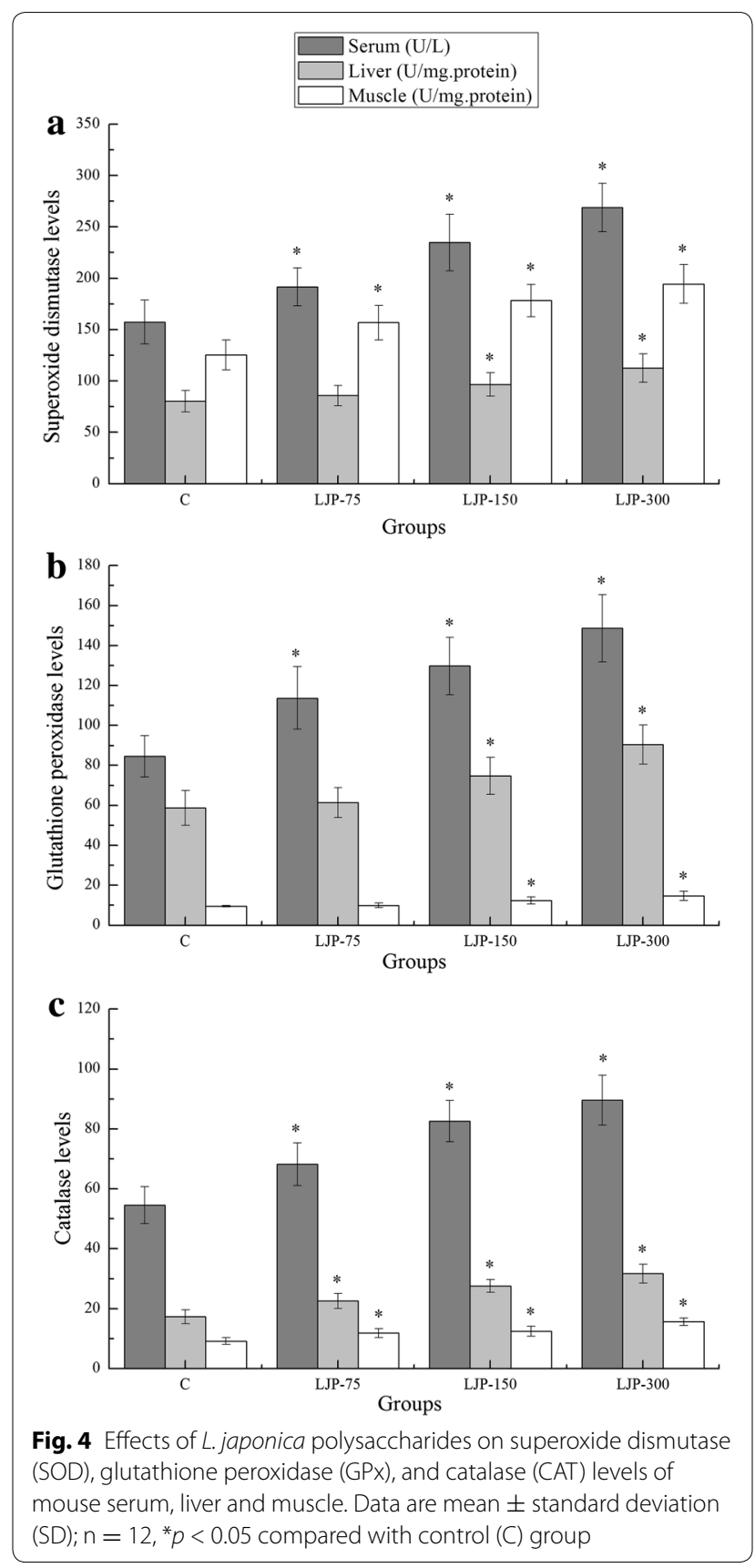


and muscle levels of the LJP-75, LJP-150, and LJP-300 groups were significantly higher than that of the $\mathrm{C}$ group $(p<0.05)$ with serum increased rates of $21.73,49.10$, and $70.81 \%$, respectively, and muscle increased rates of 24.88 , 42.05 , and $54.87 \%$, respectively. Furthermore, the SOD levels in the liver of the LJP-150 and LJP-300 groups were significantly higher than that of the $C$ group $(p<0.05)$, with increased rates of 20.09 and $40.02 \%$, respectively. Although the liver SOD level of the LJP-75 group also increased, no significant difference was observed $(p>0.05)$. As shown in Fig. $4 \mathrm{~b}$, the serum GPx levels of the LJP-75, LJP-150, and LJP-300 groups were significantly higher than that of the $C$ group $(p<0.05)$, with increased rates of $34.44,53.44$, and $75.82 \%$, respectively. In addition, the liver and muscle GPx levels of the LJP150 and LJP-300 groups were significantly higher than that of the $\mathrm{C}$ group $(p<0.05)$. Moreover, the increase rates for the LPJ-75 and LPJ-150 groups were 27.29 and $53.91 \%$, respectively in the liver, while the muscle rates were 30.87 and $55.81 \%$, respectively. Although the liver and muscle GPx levels of the LJP-75 group also increased, no significant difference was observed $(p>0.05)$. As shown in Fig. 4c, the serum, liver, and muscle CAT levels of the LJP-75, LJP-150, and LJP-300 groups were significantly higher than that of the $\mathrm{C}$ group $(p<0.05)$. Moreover, the increased ratios in the serum were $25.15,51.37$ and $64.26 \%$, respectively; in the liver, $30.35,58.92$, and $83.04 \%$, respectively; and in the muscle $28.89,35.61$, and $70.25 \%$, respectively. The results indicate that LJP upregulated the main antioxidant enzyme activity, which might protect against exhaustive exercise-induced oxidative stress. However, further research needs to be carried out to elucidate this hypothesis.

\section{Effects of LJP on malondialdehyde (MDA) content of mouse serum, liver and muscle}

Strenuous physical exercise increases the production of ROS, which consequently attack the membrane lipids and results in lipid peroxidation product formation. Significant increases in lipid peroxidation products in the serum, liver, and muscle after exhaustive exercise have been recorded in several studies [37]. Malondialdehyde (MDA), one of the final products of polyunsaturated fatty acid peroxidation, has been widely investigated in exercise studies as a marker of oxidative stress [38]. As shown in Fig. 5, the MDA content of the serum, liver, and muscle of the LJP-75, LJP-150, and LJP-300 groups were significantly lower than that of the $C$ group $(p<0.05)$. Moreover, the decreased rates in the serum were 17.63, 29.16 , and $34.68 \%$, respectively; in the liver were 13.39 , 17.23 , and $21.54 \%$, respectively; and in the muscle were $13.24,26.95$, and $60.09 \%$, respectively. These results indicate that LJP effectively reduced lipid peroxidation.

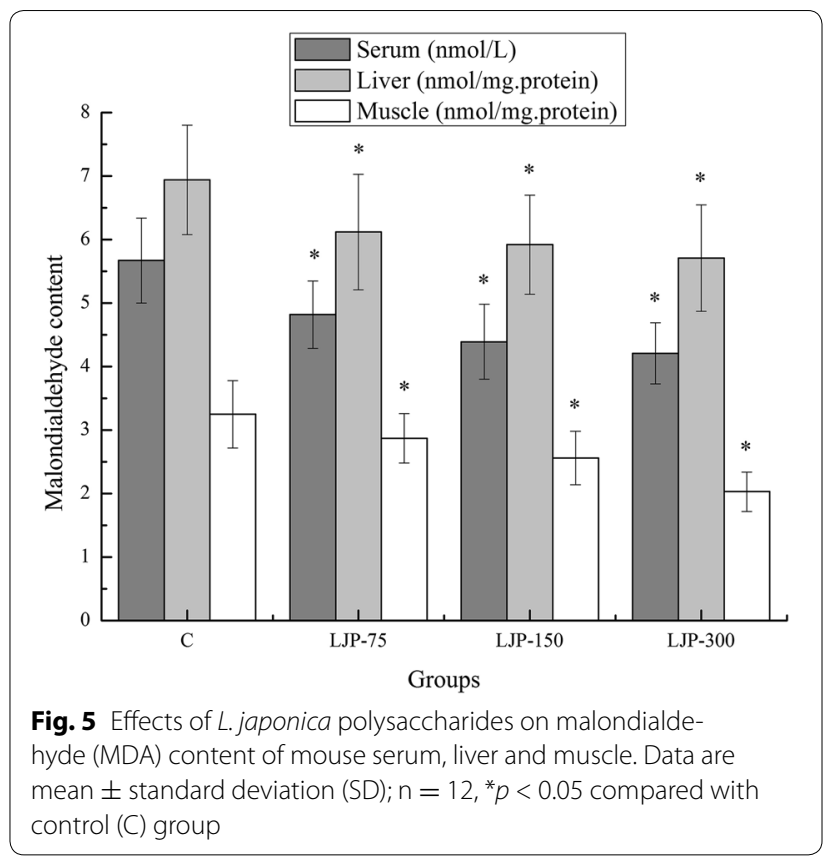

\section{Effects of LJP on serum myeloperoxidase (MPO) levels of mice}

Exercise induces neutrophil priming for oxidative activity as evidenced by the increased neutrophil myeloperoxidase (MPO) activity [39]. Accumulating evidence indicates that neutrophil infiltration into tissues is associated with exhaustive exercise-induced oxidative damage [40, 41]. Neutrophils are capable of further generating free radicals via the action of nicotinamide adenine dinucleotide phosphate oxidase [42]. In addition, neutrophils produce hypochlorite from hydrogen peroxide $\left(\mathrm{H}_{2} \mathrm{O}_{2}\right)$ by the action of MPO, a marker for neutrophil infiltration into tissues, during the induction of exercise-induced oxidative damage $[43,44]$. As shown in Fig. 6, the serum MPO levels of the LJP-75, LJP-150, and LJP-300 groups were significantly lower than that of the $C$ group $(p<0.05)$, and the decreased rates were $17.69,34.80$, and $40.54 \%$, respectively. These results indicate that LJP played an important role in inhibiting oxidative damage after exhaustive exercise, and the decreased serum MPO levels may be due to an alteration in the intracellular redox status of the neutrophils [41].

\section{Conclusions}

This study demonstrated that LJP enhanced the exercise endurance of mice by increasing swimming time to exhaustion and the glycogen contents of the liver and muscle, as well as decreasing BLA levels. Furthermore, LJP exhibited a protective effect against exhaustive exercise-induced oxidative stress by increasing the 


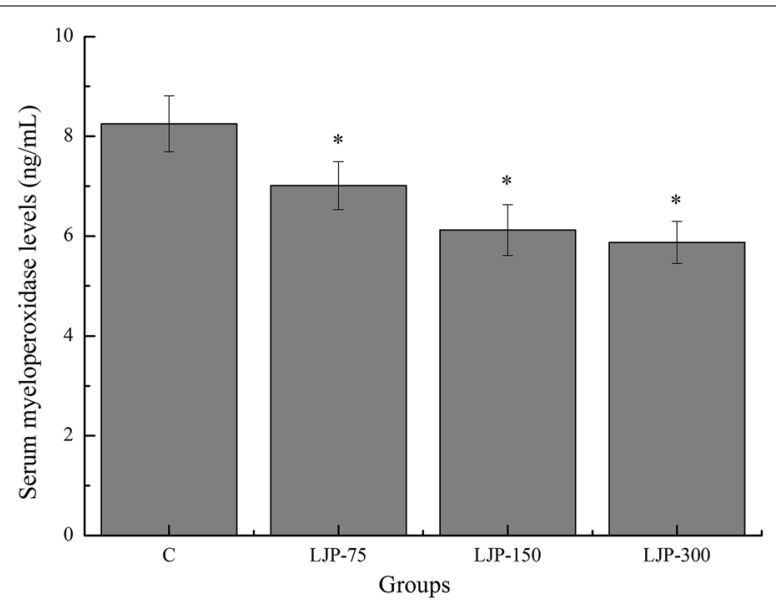

Fig. 6 Effects of L.japonica polysaccharides on serum myeloperoxidase (MPO) levels of mice. Data are mean \pm standard deviation (SD); $\mathrm{n}=12,{ }^{*} p<0.05$ compared with control (C) group

serum, liver, and muscle levels of SOD, GPx, and CAT, as well as decreasing serum MPO and the MDA contents of the serum, liver, and muscle. The experimental data have shed some light on the clinical therapeutic potential of LJP against exhaustive exercise-induced oxidative stress. However, further study is required to ascertain the detailed underlying mechanisms of these effects.

\section{Methods}

\section{Materials}

Laminaria japonica was collected in Zhoushan, Zhejiang, China in September 2013 and the plant material was identified by Professor M. J. Wang (College of Life Sciences, China Jiliang University, Hangzhou, China). The fresh $L$. japonica samples were immediately washed with water, sun-dried, ground into a fine powder using a mechanical grinder (FZ102, Taisite Instrument Co., Tianjin, China), filtered through a 40 -mesh $(200-\mu \mathrm{m})$ sieve, and then the dried powder was stored at room temperature $\left(20 \pm 2{ }^{\circ} \mathrm{C}\right)$ in a desiccator $(300 \mathrm{~mm}$, Huaou Industrial Co., Yancheng, China) until further used.

\section{Chemicals and reagents}

Purchased commercial diagnostic kits were used for the determination of BLA (Leadman Biochemistry Technology Co., Ltd., Beijing, China); tissue glycogen, SOD, GPx, and CAT (Jiancheng Biotechnology Institute, Nanjing, China); MDA (Biosino Bio-technology and Science Inc., Beijing, China); and MPO (Jianglai Biochemistry Technology Co., Ltd., Shanghai, China). All other chemicals and reagents were of analytical grade purity, and they were purchased from Hangzhou Chemical Reagent Co.,
Ltd. (Hangzhou, China), and were used without further purification.

\section{Preparation of LJP}

The LJP was prepared according to previously published method [21, 45] with minor modifications. Briefly, dried powder sample was defatted with anhydrous ethanol at $60{ }^{\circ} \mathrm{C}$ for $3 \mathrm{~h}$ with stirring and then mixed with distilled water thrice $(1: 40, \mathrm{w} / \mathrm{v})$ at $90^{\circ} \mathrm{C}$ for $2.5 \mathrm{~h}$. The insoluble residue was separated from the aqueous extract by centrifugation $(10,640 \times g$ for $15 \mathrm{~min})$. Then, the combined supernatants were concentrated to a quarter of the original volume by evaporation and deproteinated using the Sevag method [46]. The solution was added to anhydrous ethanol to obtain an ethanol concentration of $80 \%$, kept overnight, and then filtered. The resulting precipitate was dissolved in water followed by the addition of anhydrous ethanol to a final ethanol concentration of $80 \%$ and then filtered twice. The precipitate was washed sequentially with $95 \%$ ethanol, anhydrous ethanol, and acetone and then lyophilized to obtain the final extract of LJP at a yield of $21.37 \%(\mathrm{w} / \mathrm{w})$ of the original L. japonica plant material. The dried LJP was dissolved in saline solution just before use.

\section{Experimental animals}

Adult male Kunming mice (Mus musculus, Km, with weight $20 \pm 2 \mathrm{~g}$ ) were procured from the Experimental Animal Center of Zhejiang Province. All animals were housed under standard environmental conditions (temperature $21 \pm 2{ }^{\circ} \mathrm{C}$; humidity $45 \pm 5 \%$; and 12 -h light:dark cycle) with free access to a standard pellet diet and water ad libitum. All animal studies were performed according to the Guide for the Care and Use of Laboratory Animals of the National Institutes of Health (NIH), as well as the guidelines of the Animal Welfare Act. The experimental protocol was approved (approval number: ZJSR2014-0113) by the Institutional Animal Care and Use Committee (IACUC) at the Zhejiang Shuren University.

\section{Experimental design}

The mice were allowed to acclimatize to the laboratory environment for 1 week prior to the experiments. Then, the animals were assigned randomly to four groups of 12 mice each namely the C, LJP-75, LJP-150, and LJP-300 groups, which were treated with the vehicle (physiological saline) and 75,150 , and $300 \mathrm{mg} \mathrm{kg}^{-1}$ of LJP, respectively, for 28 days. The LJP was dissolved in $1.5 \mathrm{~mL}$ of the vehicle, and the $C$ group received the same volume of the vehicle as well. The treatments were administered orally by gavage once a day according to the pretest and dose determined during the active screening. 
After the final LJP or vehicle treatment, the mice were allowed to rest for $30 \mathrm{~min}$, and then they were subjected to the forced swimming test using the method described by $\mathrm{Li}$ et al. [11]. The apparatus used was an acrylic plastic pool $(60,50$, and $50 \mathrm{~cm}$ in length, width, and height, respectively) filled with fresh water, which was maintained at $25 \pm 0.5{ }^{\circ} \mathrm{C}$ at a depth of $40 \mathrm{~cm}$. Each mouse was weighted using a lead wire bundle attached to the tail at $5 \%$ of the body weight. Exhaustion was determined by observing the loss of coordinated movements and failure to return to the surface within $10 \mathrm{~s}$.

\section{Biochemical analyses}

Following the forced swimming test, the mice were anaesthetized with absolute ether and the success of the anaesthesia was confirmed by verifying the absence of reflex responses to noxious stimuli. Then, the mice were euthanized by decapitation, blood samples were collected for BLA analysis, and serum was obtained by centrifugation $\left(2000 \times g, 4{ }^{\circ} \mathrm{C}, 10 \mathrm{~min}\right)$ for the SOD, GPx, CAT, MPO, and MDA analyses. After blood collection, the liver and gastrocnemius muscle tissues were quickly dissected, washed in ice-cold physiological saline, frozen in liquid nitrogen, and stored at $-70{ }^{\circ} \mathrm{C}$ for the assays of glycogen, SOD, GPx, CAT, and MDA. The measurements were performed according to the recommended procedures provided by the commercial diagnostic kits.

\section{Statistical analysis}

The data obtained were expressed as mean \pm standard deviation (SD). The results were analyzed using a oneway analysis of variance (ANOVA) followed by a post hoc Tukey's test using the statistical package for the social sciences (SPSS) software (version 15.0, SPSS Inc., Chicago, IL, USA). Values were considered significant when $p<0.05$.

\section{Abbreviations \\ LJP: Laminaria japonica polysaccharides; BLA: blood lactic acid; SOD: superox- ide dismutase; GPx: glutathione peroxidase; CAT: catalase; MDA: malondialde- hyde; MPO: myeloperoxidase.}

\section{Authors' contributions}

FY designed and planned the study and drafted the manuscript. HH performed the analyses and collected the test results. Both authors cooperated on the interpretation of the results. Both authors read and approved the final manuscript.

\section{Author details \\ 1 Public PE Department, Zhejiang Shuren University, No. 8 Shuren Street, Hangzhou 310015, Zhejiang Province, People's Republic of China. ${ }^{2}$ Depart- ment of Physical Education and Military Training, China Jiliang University, Hangzhou 310018, China.}

\section{Acknowledgements}

We are grateful to Dr. Lijun Li (Jilin Agricultural University, China) for his revision and comments on the manuscript. This study was funded by a Grant (No.
ZGT201124) from the Association of Higher Education of Zhejiang Province, China.

\section{Competing interests}

The authors declare that they have no competing interests.

Received: 21 September 2015 Accepted: 13 April 2016

Published online: 26 April 2016

\section{References}

1. Toldy A, Stadler K, Sasvári M, Jakus J, Jung KJ, Chung HY, et al. The effect of exercise and nettle supplementation on oxidative stress markers in the rat brain. Brain Res Bull. 2005;65:487-93.

2. Sacheck JM, Blumberg JB. Role of vitamin E and oxidative stress in exercise. Nutrition. 2001:17:809-14.

3. Margaritis I, Palazzetti S, Rousseau AS, Richard MJ, Favier A. Antioxidant supplementation and tapering exercise improve exercise-induced antioxidant response. J Am Coll Nutr. 2003:22:147-56.

4. Chen Z, Li S, Wang X, Zhang CL. Protective effects of Radix pseudostellariae polysaccharides against exercise-induced oxidative stress in male rats. Exp Ther Med. 2013;5:1089-92.

5. Morales AE, Pérez-Jiménez A, Hidalgo MC, Abellán E, Cardenete G. Oxidative stress and antioxidant defenses after prolonged starvation in Dentex dentex liver. Comp Biochem Physiol C: Toxicol Pharmacol. 2004;139:153-61

6. Li X, Yu Z, Long S, Guo Y, Duan D. Hypoglycemic effect of Laminaria japonica polysaccharide in a type 2 diabetes mellitus mouse model. ISRN Endocrinol. 2012;2012:507462.

7. Morillas-Ruiz JM, Villegas García JA, López FJ, Vidal-Guevara ML, Zafrilla P. Effects of polyphenolic antioxidants on exercise-induced oxidative stress. Clin Nutr. 2006:25:444-53.

8. Voces J, Cabral de Oliveira AC, Prieto JG, Vila L, Perez AC, Duarte ID, et al. Ginseng administration protects skeletal muscle from oxidative stress induced by acute exercise in rats. Braz J Med Biol Res. 2004;37:1863-71.

9. Xu J, Li Y. Effects of salidroside on exhaustive exercise-induced oxidative stress in rats. Mol Med Rep. 2012;6:1195-8.

10. Yan F, Zhang Y, Wang BB. Effects of polysaccharides from Cordyceps sinensis mycelium on physical fatigue in mice. Bangladesh J Pharmacol. 2012;7:217-21.

11. McAnulty SR, McAnulty LS, Nieman DC, Dumke CL, Morrow JD, Utter AC, et al. Consumption of blueberry polyphenols reduces exercise-induced oxidative stress compared to vitamin C. Nutr Res. 2004;24:209-21.

12. Qi B, Zhang L, Zhang Z, Ouyang J, Huang H. Effects of ginsenosides-Rb1 on exercise-induced oxidative stress in forced swimming mice. Pharmacogn Mag. 2014;10:458-63.

13. Korivi M, Hou CW, Huang CY, Lee SD, Hsu MF, Yu SH, et al. GinsenosideRg1 protects the liver against exhaustive exercise-induced oxidative stress in rats. Evid Based Comple Altern Med. 2012;2012:932165.

14. Hao HT. Effects of Auricularia auricula polysaccharides on exhaustive swimming exercise-induced oxidative stress in mice. Trop J Pharm Res. 2014:13:1319-26.

15. Chen QP, Wei P. Icariin supplementation protects mice from exerciseinduced oxidant stress in liver. Food Sci Biotechnol. 2013;22:1-5.

16. Zhonghui Z, Xiaowei Z, Fang F. Ganoderma lucidum polysaccharides supplementation attenuates exercise-induced oxidative stress in skeletal muscle of mice. Saudi J Biol Sci. 2014;21:119-23.

17. Wang J, Zhang Q, Zhang Z, Zhang H, Niu X. Structural studies on a novel fucogalactan sulfate extracted from the brown seaweed Laminaria japonica. Int J Biol Macromol. 2010;47:126-31.

18. Zha XQ, Lu CQ, Cui SH, Pan LH, Zhang HL, Wang JH, et al. Structural identification and immunostimulating activity of a Laminaria japonica polysaccharide. Int J Biol Macromol. 2015;78:429-38.

19. Li SS, Chen ZC, Zhang CH. Effect of tao-hong-si-wutang, a traditional Chinese herbal medicine formula, on physical fatigue in mice. Afr J Tradit Comple Altern Med. 2012;10:60-5

20. Croci DO, Cumashi A, Ushakova NA, Preobrazhenskaya ME, Piccoli A, Totani L, et al. Fucans, but not fucomannoglucuronans, determine the 
biological activities of sulfated polysaccharides from Laminaria saccharina brown seaweed. PLoS ONE. 2011;6:e17283.

21. Jia X, Yang J, Wang Z, Liu R, Xie R. Polysaccharides from Laminaria japonica show hypoglycemic and hypolipidemic activities in mice with experimentally induced diabetes. Exp Biol Med (Maywood). 2014;239:1663-70.

22. Lebedynskaya EA, Makarenkova ID, Lebedynskaya OV, Akhmatova NK, Zvyagintseva TN. The effect of sulfated polysaccharides from brown seaweed Laminaria japonica on the morphology of lymphoid organs and functional characteristics of immunocompetent cells. Biomed Khim. 2014;60:581-90.

23. Lin R, Liu X, Meng Y, Xu M, Guo J. Effects of Laminaria japonica polysaccharides on airway inflammation of lungs in an asthma mouse model. Multidiscip Respir Med. 2015;10:20.

24. Wang J, Zhang Q, Zhang Z, Li Z. Antioxidant activity of sulfated polysaccharide fractions extracted from Laminaria japonica. Int J Biol Macromol. 2008;42:127-32.

25. Wang J, Zhang Q, Zhang Z, Song H, Li P. Potential antioxidant and anticoagulant capacity of low molecular weight fucoidan fractions extracted from Laminaria japonica. Int J Biol Macromol. 2010;46:6-12.

26. Xu Z, Shan Y. Anti-fatigue effects of polysaccharides extracted from Portulaca oleracea L. in mice. Indian J Biochem Biophys. 2014;5:321-5.

27. Wang SY, Huang WC, Liu CC, Wang MF, Ho CS, Huang WP, et al. Pumpkin (Cucurbita moschata) fruit extract improves physical fatigue and exercise performance in mice. Molecules. 2012;17:11864-76.

28. Zhang G, Zhou S-M, Tian J-H, Huang Q-Y, Gao Y-Q. Anti-fatigue effects of methazolamide in high-altitude hypoxic mice. Trop J Pharm Res. 2012;11:209-15.

29. Sun S, Niu H, Yang T, Lin Q, Luo F, Ma M. Antioxidant and anti-fatigue activities of egg white peptides prepared by pepsin digestion. J Sci Food Agric. 2014;94:3195-200.

30. Powers SK, Jackson MJ. Exercise-induced oxidative stress: cellular mechanisms and impact on muscle force production. Physiol Rev. 2008:88:1243-76.

31. Gündüz F, Sentürk UK, Kuru O, Aktekin B, Aktekin MR. The effect of one year's swimming exercise on oxidant stress and antioxidant capacity in aged rats. Physiol Res. 2004;53:171-6.

32. Karabulut AB, Kafkas ME, Kafkas AS, Onal Y, Kiran TR. The effect of regular exercise and massage on oxidant and antioxidant parameters. Indian J Physiol Pharmacol. 2013;57:378-83.
33. Zhou LZ, Johnson AP, Rando TA. NF kappa B and AP-1 mediate transcriptional responses to oxidative stress in skeletal muscle cells. Free Radic Biol Med. 2001;31:1405-16.

34. Mahboob M, Shireen KF, Atkinson A, Khan AT. Lipid peroxidation and antioxidant enzyme activity in different organs of mice exposed to low level of mercury. J Environ Sci Health B. 2001;36:687-97.

35. Liang FQ, Godley BF. Oxidative stress-induced mitochondrial DNA damage in human retinal pigment epithelial cells: a possible mechanism for RPE aging and age-related macular degeneration. Exp Eye Res. 2003;76:397-403.

36. Rui L. Energy metabolism in the liver. Compr Physiol. 2014;4:177-97.

37. Qi B, Liu L, Zhang H, Zhou GX, Wang S, Duan XZ, et al. Anti-fatigue effects of proteins isolated from Panax quinquefolium. J Ethnopharmacol. 2014;153:430-4.

38. Xiao N-N. Effects of resveratrol supplementation on oxidative damage and lipid peroxidation induced by strenuous exercise in rats. Biomol Ther (Seoul). 2015;23:374-8.

39. Sureda A, Tauler P, Aguiló A, Cases N, Fuentespina E, Córdova A, et al. Relation between oxidative stress markers and antioxidant endogenous defences during exhaustive exercise. Free Radic Res. 2005;39:1317-24.

40. Ji LL. Antioxidants and oxidative stress in exercise. Proc Soc Exp Biol Med. 1999;222:283-92.

41. Peake J, Suzuki K. Neutrophil activation, antioxidant supplements and exercise-induced oxidative stress. Exerc Immunol Rev. 2004;10:129-41.

42. Belviranlı M, Gökbel H. Acute exercise induced oxidative stress and antioxidant changes. Eur J Gen Med. 2006;3:126-31.

43. Lin WT, Yang SC, Tsai SC, Huang CC, Lee NY. L-Arginine attenuates xanthine oxidase and myeloperoxidase activities in hearts of rats during exhaustive exercise. Brit J Nutr. 2006;95:67-75.

44. Huang CC, Tsai SC, Lin WT. Potential ergogenic effects of L-arginine against oxidative and inflammatory stress induced by acute exercise in aging rats. Exp Gerontol. 2008:43:571-7.

45. Sheng R, Xu X, Tang Q, Bian D, Li Y, Qian C, et al. Polysaccharide of Radix pseudostellariae improves chronic fatigue syndrome induced by poly I:C in mice. Evid Based Comple Altern Med. 2011;2011:840516. doi:10.1093/ ecam/nep208.

46. Staub AM. Removal of protein-Sevag method. Methods Carbohydr Chem. 1965;5:5-6.

\section{Submit your next manuscript to BioMed Central and we will help you at every step:}

- We accept pre-submission inquiries

- Our selector tool helps you to find the most relevant journal

- We provide round the clock customer support

- Convenient online submission

- Thorough peer review

- Inclusion in PubMed and all major indexing services

- Maximum visibility for your research

Submit your manuscript at www.biomedcentral.com/submit
() Biomed Central 